\title{
Changes chemopreventive markers in colorectal cancer development after inulin supplementation
}

\author{
Hijova $E^{1}$, Szabadosova $V^{2}$, Strojny $L^{1}$, Bomba $A^{1}$ \\ Institute of Experimental Medicine, Faculty of Medicine, Safarikiensis University, Kosice, Slovakia. \\ hijova@pobox.sk
}

\begin{abstract}
Background: Natural dietary compounds such as prebiotics modulate microbial composition and could prevent the colon cancer development as potential chemopreventive agent.

Objectives: Effect of prebiotic-inulin on biochemical, microbial and chemopreventive markers were examined in Sprague-Dawley rats during experimental chemically dimethylhydrazine induced colon cancer development. Methods: Rats were divided to 3 groups: control group (CG), group with dimethylhydrazine (DMH) and group with $\mathrm{DMH}$ and prebiotic (DMH+PRE). The efficacy of the prebiotic inulin (PRE) on the activities of $\beta$-glucuronidase, short chain fatty acids (SCFAs), counts of coliforms and lactobacilli, immunoreactivity of cyclooxygenase-2 (COX-2), transcription nuclear factor kappa beta (NFKB) and inducible nitric oxide synthase (iNOS) in colon tissue were examined.

Results: Inulin significantly decreased coliforms counts $(p<0.01)$, increased lactobacilli counts $(p<0.001)$, and decreased activity of $\beta$-glucuronidase $(p<0.01)$ in fresh caecal digesta. Butyric and propionic acids concentrations were increased after inulin supplementation in comparison to $\mathrm{DMH}$ group. Application of inulin decreased immunoreactivity and numbers of COX-2, NFKB and iNOS positive cells in colon tissue in comparison to DMH group. Conclusion: Inulin suppressed expression observed markers, which play an important role in carcinogenesis and in the inflammatory process, which predisposes to the use of inulin in the prevention or treatment of inflammatory bowel disease (Tab. 1, Fig. 2, Ref. 17). Text in PDF www.elis.sk. Key words: inulin, colorectal cancer, chemoprevention.
\end{abstract}

Despite considerable progress in screening, early diagnosis and the development of noninvasive technologies colorectal cancer (CRC) remains the leading cause of mortality among men and women, not only in the world but also in Slovakia. Report on the health status of the population of the Slovak Republic for the years 2009-2011 states that after diseases of the circulatory system with a percentage of $45.9 \%$ in men and $59.8 \%$ in women are cancers in men $26.2 \%$ and women $20.1 \%$ of the second group of diseases which have the highest mortality rate in Slovakia (Report on health status of the population of the Slovak Republic, 2011). Among the risk factors for colorectal cancer include age, family history, inflammatory bowel diseases, including ulcerative colitis and Crohn's disease, procarcinogens occurring in the environment and in the food chain (Benson et al, 2007). Unexplained exact mechanism of formation of colorectal carcinogenesis, current

${ }^{1}$ Institute of Experimental Medicine, Faculty of Medicine, Safarikiensis University, Kosice, and ${ }^{2}$ Faculty of Humanities and Natural Sciences, Universiy of Presov in Presov, Presov, Slovakia

Address for correspondence: E. Hijova, MVDr, PhD, Institute of Experimental Medicine, Faculty of Medicine, LF UPJS, SNP 1, SK-040 11 Kosice, Slovakia.

Phone: +421.55 .2343463 , Fax: +421.55 .6420253$

Acknowledgements: This work was supported by grant VEGA 1/0372/10 and the Agency of the Slovak Ministry of Education for the Structural Funds of the EU, under ITMS 26220120058 (20\%), ITMS 26220220104 (20\%), ITMS $26220220152(20 \%)$. treatment options, including surgery, chemotherapy, radiotherapy, and molecularly-targeted are still limited to advanced tumors. Tumor formation - tumorigenesis in different organs is a complex multistep sequence number of accompanying symptoms such as chronic inflammation, genetic and molecular chenges, including metabolic processes thereby providing an attractive model to investigate the potential of food substances in the prevention and control of colorectal cancer through chemopreventive strategies (Pan and Ho, 2008).

A realistic approach to reduce the global burden of colorectal cancer chemoprevention is considered, where in addition to the development of synthetic materials, a wide variety of natural food compounds gives hope for chemoprevention. Chemoprevention is defined as the use of natural dietary compounds and/or synthetic substances that can delay, prevent, or even reverse the development of adenomas, as well as the progression from adenoma to carcinoma. The ultimate goal of chemoprevention by natural dietary compounds is the reduction of $\mathrm{CRC}$ incidence by intervening development pathways in tumor cells which promote growth and metastases of CRC. In this regard prebiotics as dietary natural ingredients improving intestinal function and ensuring a healthy gastrointestinal tract environment and an attract a great deal of interest. Dietary modulation of intestinal microflora by prebiotics creates a benefit which prevent the development of chronic disesases.

The aim of presented study was to obtain information about the efficacy of the prebiotic inulin on the activities of $\beta$ - gluc- 
uronidase, short chain fatty acids (SCFAs), counts of coliforms and lactobacilli, and chemopreventive markers cyclooxygenase-2 (COX-2), transcription nuclear factor kappa beta (NFKB) and inducible nitric oxide synthase (iNOS) in rats colon tissue with dimethylhydrazine induce colon cancer development.

\section{Material and methods}

Animals

Male and female Sprague-Dawley rats four months old with mean initial body weight $378.73 \pm 81.25 \mathrm{~g}$ were housed and kept under cycles of 12 hours light to 12 hours dark. Experiment was conducted in accordance with the principles of the Slovak Republic for the Care and Use of Laboratory Animals and approved by the Ethical Committee of the Faculty of Medicine of P. J. Šafarik University in Košice. Rats were randomly assigned to 3 groups (10 per group): CG control group (with conventional feed), DMH group (received dimethyhydrazine-DMH, Merck, Germany at the dose of $21 \mathrm{mg} / \mathrm{kg}$ body weight subcutaneously five times with weekly interval and conventional feed) and DMH+PRE group (received conventional feed, DMH and oligofrucose-enriched inulin, BeneoSynergy 1, ORAFTI, Tienen, Belgium at the dose of $80 \mathrm{~g} /$ $\mathrm{kg}$ of conventional feed). After 7 months duration of experiment rats were euthanized under anesthesia (Ketamin $100 \mathrm{mg} / \mathrm{kg}+\mathrm{Xy}-$ lazin $15 \mathrm{mg} / \mathrm{kg}$ body weight, intraperitoneal), taken fresh faecal samples and samples of rats colon tissue were used for microbial, biochemical and immunohistochemical analysis.

\section{Microbial analyses}

Total lactobacilli and coliforms of the faecal samples were carried out after the completion of the experiment. Faeces (1g) was placed in a sterile polyethylene Stomacher Lab Blender bag with $9 \mathrm{ml}$ sterile $0.9 \% \mathrm{NaCl}$. Series of 10 -fold dilutions $\left(10^{-2}\right.$ to $\left.10^{-8}\right)$ were made in the same sterile diluent. From appropriate dilution, $0.1 \mathrm{ml}$ aliquots were spread onto two selective Mc Conkey agar (Merck, Germany) for coliforms and Rogosa agar (Biocar diagnostic, France) for lactobacilli. The plates for lactobacilli were made anaerobic (Gas PaK, USA) and incubated at $37^{\circ} \mathrm{C}$ for 48 hours and for coliforms incubated aerobically at $37^{\circ} \mathrm{C}$ for $16-18$ hours. The viable counts are expresses as the $\log 10$ of colony forming units (CFU) per gram of faeces.

\section{Biochemical analysis}

The activity of $\beta$-glucuronidase in fresh caecal digesta was measured by the rate of $p$ - or $o$ - nitrophenol from their nitrophenylglucosides according to the method described by Juśkiewicz (2002). The SCFAs were analysed in the caecal digesta using gas chromatography (Hewlett Packard 6890 Plus, USA) and was expressed as $\mathrm{mmol} / 100 \mathrm{ml}$ of wet cecal digesta.

Immunohistochemical analysis of chemopreventive marker COX2, NF $\kappa B$ and $i N O S$ in colon tissue

Immunohistochemical staining of markers $\mathrm{COX}-2, \mathrm{NF} \kappa \mathrm{B}$ and iNOS in colon tissue was performed on paraffin sections of 4-5 $\mu \mathrm{m}$ using a commercial kit HRP-DAB Cell \& Tissue Staining Kit
(R\&D Systems, UK). Immunoreactivity in the tunica mucosae and in tela submucosae of colon was localized by immunohistochemistry using specific antibodies anti-COX-2 (diluted 1:500; Abcam, UK), anti-NFкB (diluted 1:100; MBL International, USA) and anti-iNOS (diluted 1:5000; Sigma Aldrich, Czech Republic). Immunoreactivity of COX-2, NFאB and iNOS was expressed as the total number (average of tunica mucosae and tela submucosae) of positive cells calculated to the area of $1000 \mu \mathrm{m}^{2}$ tissue of the colon.

\section{Statistical analysis}

Results are expressed as mean \pm SD. Statistical analysis was performed using ANOVA with p values $(<0.05)$ were considered to be statistically significant.

\section{Results}

The mean body weight of the rats at the end of the experiment in the CG group was increased by $21.43 \%$, by $19.89 \%$ in $\mathrm{DMH}$ group, and by $28 \%$ in DMH+PRE group. In the control group the count of coliform was $6.17 \pm 0.56 \log _{10} \mathrm{CFU} / \mathrm{g}$ and of lactobacilli $8.99 \pm 0.45 \log _{10} \mathrm{CFU} / \mathrm{g}$. DMH injection nonsignificantly increased coliforms and decreased lactobacilli counts $(6.34 \pm 0.25$; $8.78 \pm 0.37$, respectively). Inulin significantly decreased coliforms counts $\left(5.96 \pm 0.22 \log _{10} \mathrm{CFU} / \mathrm{g} ; \mathrm{p}<0.01\right)$ and significantly increased lactobacilli counts $\left(9.38 \pm 0.29 \log _{10} \mathrm{CFU} / \mathrm{g} ; \mathrm{p}<0.001\right)$ in comparison to DMH group. The changes in activity of $\beta$-glucuronidase in all groups are showed in Figure 1. DMH injection increased activity of $\beta$-glucuronidase nonsignificantly but prebiotic supplementation decreased their activity $(\mathrm{p}<0.01)$. Butyric and propionic concentrations were decreased in DMH group $(\mathrm{p}<0.001)$, but prebiotic positively increased its concentration $(p<0.01)$, (Table 1$)$. The provided proof is finding the highest total numbers of COX-2 positive cells in colon tissue in DMH group with induced colon cancer development (Fig. 2). Similar tendency changes in DMH

Tab. 1. Concentration of short chain fatty acids in different groups.

\begin{tabular}{lccc}
\hline Groups & $\begin{array}{c}\text { Acetate } \\
(\mathrm{mmol} / 100 \mathrm{ml})\end{array}$ & $\begin{array}{c}\text { Propionate } \\
(\mathrm{mmo} / 100 \mathrm{ml})\end{array}$ & $\begin{array}{c}\text { Butyrate } \\
(\mathrm{mmol} / 100 \mathrm{ml})\end{array}$ \\
\hline CG & $70,16 \pm 9,63$ & $12,24 \pm 1,14$ & $9,87 \pm 1,12$ \\
DMH & $67,10 \pm 5,63$ & $7,68 \pm 0,65^{* * *}$ & $6,40 \pm 0,76^{* * *}$ \\
DMH+PRE & $71,66 \pm 7,84$ & $13,21 \pm 1,87^{* * *}$ & $15,03 \pm 2,04^{* * *}$ \\
\hline
\end{tabular}

Statistical significance is between $\mathrm{CG} / \mathrm{DMH}$ and $\mathrm{DMH} / \mathrm{DMH}+\mathrm{PRE} * * * \mathrm{p}<0.001$.

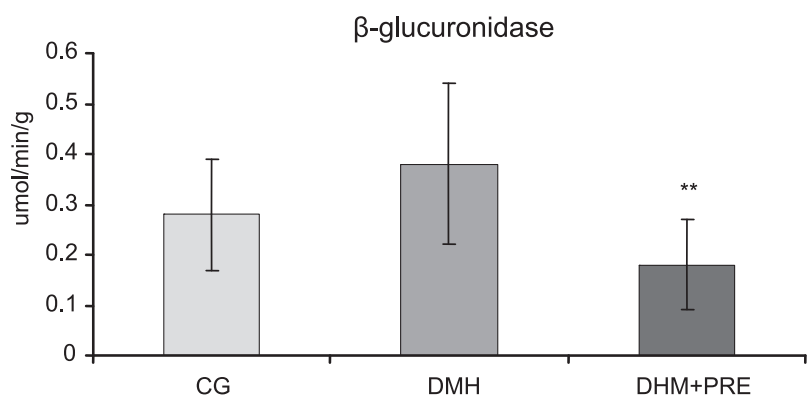

Fig. 1. Changes in activity of $\beta$-glucuronidase. Statistical significance is between CG/DMH and DMH/DMH+PRE ** $p<0.015$. 


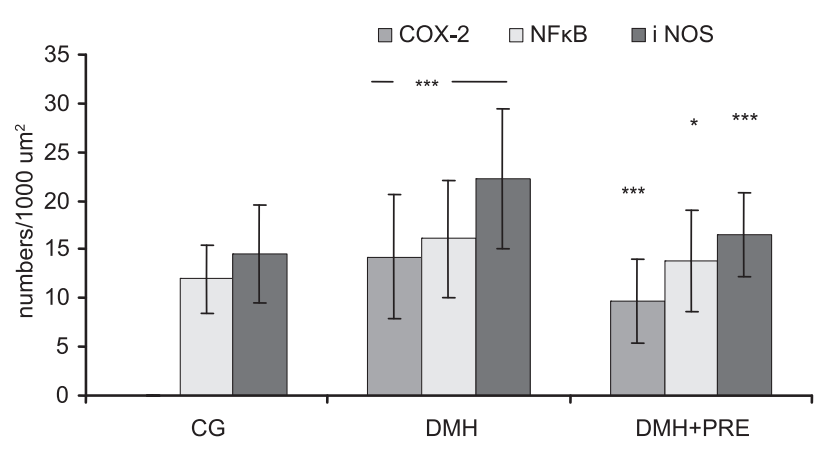

Fig.2 COX-2, NFkB and iNOS total numbers of positive cells in colon tissue. Statistical significance is between CG/DMH and DMH/ $/$ DMH+PRE * $\mathbf{p}<0.05 ; * * * \mathbf{p}<0.001$.

group were recorded in total numbers of NFKB and $\mathrm{NOS}$ positive cells in colon tissue $(p<0.001)$. Applicated inulin decreased the total numbers of positive cells COX-2, NFkB and iNOS (Fig. 2).

\section{Discussion}

Inulin prebiotic belongs to the class of non.digestible oligosacharides known as fructans (Gibson et al, 2004). To be effective prebiotics must escape digestion in the upper gastrointestinal tract and the lower part is used by beneficial microorganisms colon mainly bifidobacteria and lactobacilli. Intestinal microflora is involved in the etiology of colorectal cancer, but the exact type of bacteria associated with its occurence is not understood, but it is clear that certain groups of bacteria (lactobacilli and bifidobacteria) have a much lower activity of enzymes that can generate carcinogenic than other species such as coliforms, clostridia and Bacteroides. In our experimental work the highest number of lactobacilli was in the group where was applied inulin. The number of coliforms was in this group significantly reduced, whereas in the DMH group was highest. Activity of the enzyme $\beta$-glucuronidase is considered as a biomarker of increased risk incidence of neoplasms (Juśkiewicz et al, 2009) and at the same time is perceived as harmful to health as a result of release properties of carcinogens from glucuronic acid conjugates and critical factor enterohepatic circulation of drugs and other compounds. Bacterial fermentation of undigested food in the colon by microorganisms produce different microbial metabolites (Wong et al, 2006). The major metabolites are short chain fatty acids (SCFAs) mainly acetic acid, propionic and butyric acid. The molar ratio of acetic acid to propionic and butyric acid is between 48:29:23 and 70:15:15, rspectively, on average this ratio is given as 60:20:20. The greater part of the literature focuses on butyric acid and acetic acid, less on propionic consequently their potential effects on the physiology and pathology are underestimated (Al-Lahham et al, 2010).

Butyrate causes apoptosis, reduces metastasis in colon cell lines and protects against genotoxic carcinogens (Scharlau et al, 2009). Increased concentration of acetic acid in the DMH+PRE group is consistent with the experimental animal model point to the fact that inulin has an anti-carcinogenic effect (Pool-Zobel and Sauer, 2007). Propionic acid is produced by fermentation of polysaccharides, oligosaccharides, proteins, peptides, and glycoprotein precursor anaerobic intestinal microflora, but in quantitative terms undigested carbohydrates are the main source of production of propionic acid.

It is well known that the gastrointestinal tract is still at the stage of low-grade inflammation. Dietary fiber intake in food, which is the primary substrate for the production of propionic acid is associated with a reduction in low-grade inflammation and the pathogenesis of intestinal inflammation (Galisteo et al, 2008). Propionic acid has a slight inhibitory effect on the activity of cyclooxygenase, an anti-inflammatory effect of propionic acid mediated by cyclooxygenase may be one of the mechanisms for reducing the inflammation of the intestinal mucosa by applying prebiotic diet. Then prebiotic diet and cyclooxygenase inhibition is associated with reduction in the incidence of colorectal cancer (Comalada et al, 2006). Cyclooxygenase is an important enzyme involved in inflammatory processes. Increased expression of COX2 but not COX-1 has been observed in various types of tumors and abnormal cells (Sano et al, 1995). The amount of COX-1 protein in the cells is practically constant such a physiological as well as pathological conditions. On the other hand, COX-2 is inducible, barely detectable under normal physiological condition (Fig. 2) but its level rises rapidly and transiently as one of the first response to proinflammatory mediators, including cytokines, mitogenic stimulus, endotoxins, growth factors and oncogenes. Supplementation of inulin in the diet reduced the number of COX-2 positive cells which pointed to its anti-inflammatory effect (Schley and Field, 2002). Recent studies have demonstrated that a transcription nuclear factor kappa beta is needed in the regulation of COX-2 (Bakhle, 2001). Chemopreventive phytopharmacs have an inhibitory effect on COX-2 suitable blocking NFkB activation. NFkB transcriptional activity is regulated through a series of intracellular signal transduction processes in reponse tovarious external stimuli and inflammatory cytokine (Chen et al, 1999). Not only the number of COX-2 positive cells in the colon tissue, but also the total numer of NFkB and iNOS was significantly reduced after administration of inulin in the diet. Increased expression of iNOS is induced positive regulation of gene probably NOS2, which codes for iNOS. Femia (2010) showed in their experiment lasting 13 weeks on the positive effect of arabinoxylan-oligosaccharides in reducing preneoplastic changes.

\section{Conclusion}

The results of our experiment have shown that inulin reduced preneoplatic changes and signs of inflammation, decreased the expression of COX-2, iNOS and NFkB. By determinig of the chemopreventive markers COX-2, iNOS and NFkB which can be characterized as inflammatory markers, because they are involved in the inflammatory process, we confirmed the presence of inflammation in the colon where the number of COX-2, NFkB and iNOS immunoreactive cells was significantly higher after DMH applications than in the control group. Chronic inflammation is associated with malignancy and is one of the main causes of various cancers as well as the development of colorectal cancer. The positive ef- 
fect of inulin was reflected in an increase in lactobacilli as well as the concentration of short chain fatty acids and by reducing the activity of $\beta$-glucuronidase. Inulin suppressed expression observed markers, which play an important role in carcinogenesis and in the inflammatory process, which predisposes inulin for use in the prevention or treatment of chronic diseases.

\section{References}

1. Bakhle YS. COX-2 and cancer: a new approach to an old problem. $\mathrm{Br}$ J Pharmacol 2001; 134: 1137-1150.

2. Benson AB. 3rd Epidemiology, disease progression, and economic burden of colorectal cancer. J Manag Care Pharm 2007; 13: S5-S18.

3. Comalada M, Bailon E, de Haro O, Lara-Villoslada F, Xaus J, Zarzuelo A, Gálvez J. The effects of short-chain fatty acids on colo epithelial proliferation and survival depend on the cellular phenotype. J Cancer Res Clin Oncol 2006; 132: 487-497.

4. Chen F, Castranova V, Shi X, Demers LM. New insights into the role of nuclear factor $-\mathrm{\kappa B}$, a ubiquitous transcription factor in the iniciation of diseases. Clin Chem 1999; 45: 7-17.

5. Femia AP, Salvadori M, Broekaert WF, François IEJA, delcour JA, Courtin CM, Caderni G. Arabinoxylan-oligosaccharides (AXOS) reduce preneoplastic lesions in the colon of rats with 1,2-dimethylhydrazine (DMH). Eur J Nutr 2010; 49: 127-132.

6. Galisteo M, Duarte J, Zarzuelo A. Effects of dietary fibers on disturbances clustered in the metabolic syndrome. J Nutr Biochem 2008; 19: 71-84.

7. Gibson GR, Probert HM, Loo VJ, Rastall RA, Roberfroid MB. Dietary modulation of the human colonic microbiota:updating the concept of prebiotics. Nutr Res Rev 2004; 17: 259-275.

8. Juśkiewicz J, Zduńczyk Z, Wróblewska M, Oszmiański J, Hernandez T. The responce of rats to feeding with diets containing grapefruit flavonoid extract. Food Res Int 2002; 35: 201-205.
9. Juśkiewicz J, Wróblewska M, Jaroslawska J, Baliński P, Matusevičius P, Zduńczyk P, Biedrzycka E, Zduńczyk Z. Effects of inulin supplemented to cellulose-free or cellulose-rich diets on caecal environmental and biochemical blood parameters in rats. J Anim Feed Sci 2009; 18: 709-722.

10. Al-Lahham AH, Peppelenbosch MP, Roelofsen H, Vonk RJ, Venema K. Biological effects of propionic acids in humans,; metabolism, potential applications and underlying mechanisms. Biochim Biophys Acta 2010; 1801: 1175-1183.

11. Pan MH, Ho CT. Chemopreventive effects of natural dietary compounds on cancer development. Chem Soc Rev 2008; 37: 2558-2574.

12. Pool-Zobel BL, Sauer J. Overview of experimental data on reduction of colorectal cancer risk by inulin-type fructans. J Nutr 2007; 137 : 2580S-2584S.

13. Report on health status of the population of the Slovak Republic 2009-2011.Office of Public Health SR and National Health Information Center, Bratislava 2011, 72 p.

14. Sano H, Kawahito Y, Wilder RL,Hashiramoto A, Mukai S, Asai K, Kimura S, Kato H, Kondo M, Hla T. Expression of cyclooxygenase-1 and -2 in human colorectal cancer. Cancer Res 1995; 55: 3785-3789.

15. Scharlau D, Borowicki A, Habermann $\mathbf{N}$ et al. Mechanisms of primary cancer prevention by butyrate and other products formed during gut flora-mediated fermentation of dietary fibre. Mutat Res Rev Mutat Res 2009; 682: 39-53.

16. Schley PD, Field CJ. The immune-enhancing effect od fietary fibres and prebiotics. Br J Nutr 2002; 87: 221-230.

17. Wong JM, deSouza R, Kendall CW, Emam A, Jenkins DJ. Colonic health: fermentation and short chain fatty acids. J Clin Gastroenterol 2006; 40: 235-243.

Received January 16, 2014. Accepted January 20, 2014. 